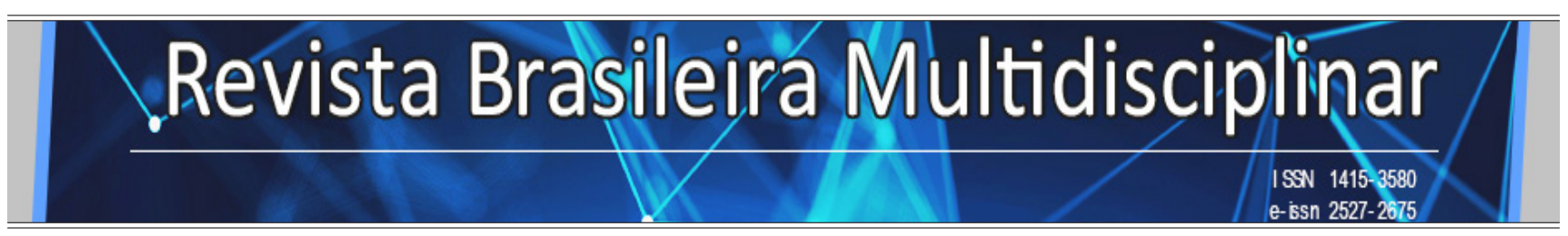

http://revistarebram.com/index.php/revistauniara

\title{
ATENDIMENTO de HIPERTENSÃo ARTERIAL SISTÊMiCA NA ESTRATÉGIA SAÚDE DA FAMÍlia: SOB A ÓtiCA DE ENFERMEIROS E AGENTES COMUNITÁRIOS DE SAÚDE
}

Joice Fernanda de Souza Santos*; Aline Natália Domingues**; Adriana Aparecida Mendes ${ }^{* * *}$; Silvia Helena Tognoli ${ }^{* * *}$; Márcia Niituma Ogata ${ }^{* * * *}$; Cibele Correia Semeão Binotto ${ }^{* * * * *}$

* Enfermeira formada pela Universidade de Araraquara. UNIARA.

** Doutoranda pela Escola de Enfermagem de Ribeirão Preto da Universidade de São Paulo.

*** Doutora em Ciências pelo Programa de Pós-Graduação em Enfermagem em Saúde Pública pela Escola de Enfermagem de Ribeirão Preto da Universidade de São Paulo.

${ }_{* * * *}$ Doutoranda do Programa de Enfermagem Fundamental da Escola de Enfermagem de Ribeirão Preto da Universidade de São Paulo.

${ }_{* * * * *}$ Pós doutora pela faculdade de enfermagem da universidade estadual do Rio de Janeiro.

****** Doutoranda do Programa de Pós Graduação da Universidade Federal de São Carlos.

*Autor para correspondência e-mail: cibelec_s@yahoo.Com.Br

\section{Palavras-chave}

Ensino de literatura

Metodologias de leitura

Leitura de clássicos

\section{KEYWORDS}

Literature Teaching

Reading Iethodologies

Reading Classics

\begin{abstract}
RESUMO
O objetivo desta pesquisa foi identificar o fluxo de atendimento e acompanhamento do paciente hipertenso no âmbito da Estratégia Saúde da Família no interior do estado de São Paulo. Trata-se de uma pesquisa qualitativa, de caráter exploratório e transversal, realizada em duas unidades de Estratégia Saúde da Família. Os dados foram coletados por meio de uma única entrevista gravada utilizando um roteiro composto por cinco questões semiestruturadas para enfermeiros e agentes comunitários de saúde, no qual participaram do estudo 12 profissionais, sendo 3 enfermeiros e 9 agentes comunitários de saúde. As entrevistas foram transcritas e analisadas segundo proposta do método de análise temática. Os resultados revelaram categorias que mostram atividades que buscam organizar, programar e planejar a assistência e manejo do usuário portador de hipertensão arterial sistêmica. Conclui-se que o fluxo de atendimento do portador de hipertensão arterial sistêmica na Estratégia Saúde da Família não se limita apenas a consultas pontuais ou tratamento medicamentoso, pois entre os desafios relacionados ao manejo adequado do usuário, se destaca a organização dos serviços de saúde em suas ações de gestão, planejamento e enfrentamento de Doenças Crônicas Não Transmissíveis.
\end{abstract}

CARE TO PATIENTS WITH SYSTEMIC ARTERIAL HYPERTENSION IN THE FAMILY HEALTH STRATEGY: THE PERSPECTIVE OF REGISTERED NURSES AND COMMUNITY HEALTH AGENTS

This research aimed to identify the flow of care and follow-up of hypertensive patients within the Family Health Strategy in the interior of the state of São Paulo. This qualitative, exploratory and cross-sectional research was carried out in two Family Health Strategy units. Data were collected through a single recorded interview using a script composed of five semi-structured questions for nurses and community health agents, with participation of 12 professionals (three nurses and nine community health agents). Interviews were transcribed and analyzed using thematic analysis. Results reveale categories that show activities that seek to organize, program and plan the care and management of users with systemic arterial hypertension. It is concluded that the flow of care of patients with systemic arterial hypertension in the Family Health Strategy is not limited to specific appointments or drug treatment. Among the challenges related to the proper management of users, it is highlighted the organization of health services in its actions for management, planning and coping with non-communicable chronic diseases. 


\section{INTRODUÇÃo}

O Sistema Único de Saúde, como política pública no Brasil possui a Atenção Primária à Saúde (APS) como principal porta de entrada para o cuidado, a mesma tem como objetivo ser a ordenadora e coordenadora das redes de atenção à saúde. Na APS ocorre o processo de restruturação do cuidado incorporando novas tecnologias e propostas organizacionais como a criação da Estratégia Saúde da Família (ESF) que possui abrangência nacional (MENDES, 2015).

A ESF é uma estratégia de qualificação na APS que visa valorizar um cuidado multiprofissional, pois entende que a complexidade dos problemas atuais exige diferentes olhares para a sua resolução (MENDES, 2015).

Os Agentes Comunitários de Saúde (ACS), integrantes da ESF são considerados o elo entre a comunidade e a unidade de saúde, uma vez que possuem contato direto com a população em seu domicílio, tornando-se conhecedores das reais necessidades da população (GUIMARÃES, 2017).

Os mesmos possuem papel fundamental na assistência aos pacientes portadores de Doenças Crônicas Não Transmissíveis (DCNT), como a Hipertensão Arterial Sistêmica (HAS), pois no momento das visitas eles podem fornecer orientações a esses pacientes sobre riscos para doenças cardiovasculares e direcionamento de condutas terapêuticas, além de monitorar o comparecimento destes às consultas e realizar a busca ativa no caso de faltas (GUIMARÃES, 2017).

Por outro lado, o enfermeiro da ESF possui a responsabilidade de realizar visitas domiciliares, consultas de enfermagem, atividades de grupo, procedimentos privativos, ações de educação permanente com a equipe, além de planejar e avaliar as ações desenvolvidas pelos ACS, com o intuito de garantir a qualidade da assistência (MENDES, 2015).

As DCNT integram o conjunto de condições crônicas, que se interligam a múltiplas causas, possui evolução crescente de difícil prognóstico. A HAS é considerada um problema de Saúde Pública de ordem nacional e mundial, sendo associado à riscos para doenças cardiovasculares e cerebrovasculares. Está relacionada à $40 \%$ dos óbitos por acidente vascular cerebral e $25 \%$ dos óbitos por doença coronariana (BRASIL, 2018).

Considerando a necessidade de desenvolver um trabalho conjunto entre profissional e usuário, o Ministério da Saúde (MS) tem incentivado a busca de melhorias no atendimento, onde os profissionais se organizam para o acolhimento do usuário, avaliando o risco e a vulnerabilidade com a finalidade de identificar a conduta a ser adotada (BRASIL, 2013).

Para produzir essas novas formas de cuidado, o MS disponibiliza protocolos assistenciais, fluxogramas e escalas de avaliação de riscos, entre elas a escala de Framighan utilizada para acompanhar usuários portadores de HAS e com referência nos resultados realizar o planejamento da assistência (BRASIL, 2013).

Assim, a identificação do risco cardiovascular deve incorporar variáveis relacionadas ao estilo de vida, histórico pessoal e familiar de outras doenças crônicas, para que seja possível realizar intervenções com o objetivo de prevenção complicações (PAULA et al., 2013).

Destaca-se que a periodicidade de consultas que o paciente será submetido varia de acordo com a somatória da classificação de risco de Framingham e as necessidades individuais, sendo realizadas as consultas médicas e de enfermagem intercaladas (BRASIL, 2014).

Desta forma, na APS considerando a importância do segmento e cuidado do paciente hipertenso na ESF, se faz necessário compreender como ocorre o processo de atendimento e acompanhamento com a finalidade de promover melhorias na qualidade de vida dos portadoraes de HAS.

Assim, o objetivo desse estudo foi identificar o fluxo de atendimento e acompanhamento do paciente hipertenso na ESF.

\section{Métodos}

Trata-se de uma pesquisa qualitativa de caráter exploratório e transversal. O estudo foi realizado em 
um município no interior de São Paulo, em duas unidades de ESF, sendo uma composta por uma equipe e outra por duas equipes.

Os participantes da pesquisa foram 3 enfermeiros e 9 ACS que atenderam ao critério de inclusão tempo mínimo de 6 meses de atuação nas unidades selecionadas, excluindo-se afastados ou ausentes no dia da coleta de dados. Divididos entre as três equipes o total desses profissionais eram, 3 enfermeiros e 14 agentes comunitários.

Os dados foram coletados por meio de uma única entrevista gravada individualmente em uma sala reservada dentro da unidade de saúde, os dados foram coletados durante os meses de agosto e setembro de 2017 de acordo com roteiro semiestruturado, composto de cinco questões norteadoras que contemplavam conhecer os desafios do atendimento ao usuário hipertenso, a identificação de ações de prevenção, promoção e controle. Posteriormente as entrevistas foram transcritas e analisadas. Para garantir o anonimato os participantes foram identificados pelas siglas ENF (enfermeiros) e ACS (Agentes Comunitários de Saúde).

Foi utilizado o método de Análise Temática realizada em três etapas, sendo a primeira classificada como pré-análise, com seleção dos documentos e foco nos objetivos da pesquisa, e ainda contempla a leitura flutuante, constituição do corpus, formulação e reformulação de hipóteses e objetivos. A segunda etapa corresponde a exploração classificatória do material, possibilitando a compreensão do texto, sendo necessário encontrar categorias. Nesse processo de categorização ocorre a redução do texto às expressões ou palavras significativas das quais o conteúdo foi organizado. Na terceira etapa, o tratamento dos resultados obtidos e interpretações, pois; os dados brutos são analisados com a finalidade de colocar em destaque as informações obtidas (MINAYO, 2014).

Foram atendidos os aspectos éticos da pesquisa segundo as orientações da Resolução n 466/12 do Conselho Nacional de Saúde. Destaca-se que esta pesquisa foi aprovada pelo Comitê de Ética em Pesquisa sob o número do parecer 2.225.253 e CAAE: 68719417.4.0000.583.

\section{RESUltados}

Participaram desse estudo 12 profissionais, sendo 3 (25\%) enfermeiros e 9 (75\%) ACS que aceitaram participar do estudo e assinaram o Termo de Consentimento Livre e Esclarecido.

Após a organização e análise do conteúdo emergiram duas categorias e suas respectivas subcategorias. A primeira categoria temática foi denominada como Atendimento ao paciente de HAS na ESF, com as suas subcategorias: ações de rastreamento da hipertensão na unidade; grupos de HAS; visitas domiciliares e acompanhamento do usuário; e a segunda categoria temática intitulada como Formação do profissional de saúde, com as subcategorias: reunião de equipe e educação permanente. A seguir será apresentado as categorias:

\section{Categoria 1: Atendimetno ao paciente de HAS na ESF}

SUbCATEgoria: AÇÕES DE RASTREAMENTO DA HIPERTENSÃo ARTERIAL SISTÓLICA NA UNIDADE

Quando foi perguntado sobre como os usuários hipertensos da unidade eram identificados, os profissionais contavam sobre a rotina de trabalho:

A pessoa que vem procurar a unidade por alguma outra, outra queixa, né, no acolhimento, porque tá com dor de ouvido, ou dor na coluna, então a gente faz o rastreamento nesse paciente. Então a gente verifica a pressão pra ver quais são os níveis dele. E quando ele vem passar em outro atendimento também é... na unidade de saúde, por exemplo, vem passa em atendimento de saúde da mulher, então a gente aproveita fazer também o rastreamento (ENF1). 
Na consulta, quando passa em consulta, que a pessoa já... tá vindo aferir a pressão, depois é diagnosticada (ACS8). Tem paciente que, busca a unidade por conta de sintomatologia, por conta de alguma coisa que ele apresenta naquele momento, então a gente consegue fazer a aferição da pressão mediante queixa/conduta. No acolhimento... ou na busca desse paciente pela unidade quando a gente faz o acolhimento, sem consulta agendada (ENF2).

A busca né, de casos novos, de investigar realmente nas consultas, o histórico todo dessa pessoa, o familiar, o dele mesmo, toda história de vida, né? O exame físico, uma anamnese bem feita, como rastreamento (ENF3).

\section{SUBCATEGORIA: GRUPOS REALIZADOS NA UNIDADE}

Os profissionais de saúde afirmam que os grupos realizados na unidade são diferentes formas de ofertar um cuidado em saúde para o portador de hipertensão, e não apenas um cuidado físico, como também um cuidado terapêutico.

A gente tem alguns grupos que desenvolve isso tanto para a parte física, quanto para a mental também (ENF3). A gente faz grupos né, isso costuma ajudar bastante (ACS6).

Nós temos aqui o grupo de caminhada, né. São orientados os pacientes à atividade física (ACS8).

\section{SUBCATEGORIA: Visitas DOMICILIARES}

Destacou-se nas falas dos entrevistados a importância das visitas domiciliares como forma de acompanhamento dos usuários com hipertensão, além do papel fundamental do agente comunitário nesse processo.

Principalmente os pacientes que acompanham aqui, eles recebem visitas mensais (ACS1). A gente orienta, vai nas casas, hã... quem é hipertenso tem que ir rigorosamente todos os meses, é uma forma de acompanhar e controlar os portadores de hipertensão (ACS2).

Sim, a gente faz visita mensalmente a todos os hipertensos da área ... todo mês é feita a visita (ACS3). $E$, as principais intervenções são as... visitas mensais dos ACS (ACS4). A gente tem um controle, é... mensal, né, nosso principal papel são as visitas (ACS5).

\section{Subcategoria: ACOMpanhamento do USUÁrio}

Segundo os participantes da pesquisa, o usuário hipertenso é acompanhado rigorosamente através do controle das consultas que ocorre de forma intercalada entre o médico e enfermeiro.

O controle é feito da seguinte forma, utilizamos os critérios do Ministério, a gente acompanha o paciente dentro do protocolo de hipertenso, a cada seis meses consulta médica... tá? E a cada dois meses consulta de enfermagem, assim ele não fica desassistido (ENF2).

Aqui nós temos um controle... ah... cada ACS tem seu controle de hipertenso. Aqui na unidade, os hipertensos eles passam a casa seis meses, no... na... consulta com o doutor e depois a consulta com a enfermeira (ACS8). Agora agendamento de três ou seis meses para que o paciente não se perca, dependendo da necessidade, até menos meses (ACS6).

\section{CATEgoria 2: FormaÇÃo do PROFissional de SAÚde SUBCATEGORIA: REUNIÃO DE EQUIPE}


A respeito do momento de discussão em equipe sobre o cuidado prestado ao usuário hipertenso, dois pontos ganham destaque, a Reunião de equipe e Educação Permanente como momentos de qualificação do cuidado prestado.

Nós temos reunião de equipe, semanalmente, então ela é realizada com toda equipe né, então com o médico, enfermeiro, o dentista, auxiliares, e técnicos, os agentes comunitários è medida que vem é... surgem questões, né, relacionadas à pacientes, tanto de hipertensão como de outros, outros... problemas de saúde e aí, são definidas, as condutas de cada caso (ENF1).

Nós temos uma reunião semanal de equipe onde a gente discute os pacientes que tem hipertensão e também outros problemas de saúde, e também problemas sociais, de tudo (ACS1).

A gente tem reunião semanal, né? Todas as segundas-feiras à tarde a gente tem discussão sobre isso, sobre caso de família, né? Então aborda, discute, resolve bastante coisa com a equipe toda. A gente tira dúvidas, tenta solucionar problemas (ACS5). Nas reuniões de equipe nós discutimos alguns casos né, quando a gente sai para o campo, para visita, se a gente identifica algum problema, é colocado em reunião de equipe (ACS8).

O processo de trabalho é estruturado por intermédio das reuniões de equipe, pois durante a jornada de trabalho semanal os profissionais reúnem informações, dúvidas e conhecimentos que são levados para discussão neste momento de tamanha importância.

\section{Subcategoria: EduchęÃo Permanente}

Ficou evidente no relato de uma das enfermeiras que a abordagem sobre o cuidado com o paciente hipertenso ocorre no momento da educação permanente, é por meio dessas discussões que as condutas são estabelecidas:

Nós temos uma reunião semanal de educação é... permanente da própria equipe, que a gente chama de discussão do processo de trabalho em equipe, e... entra dentro temas como hipertensão, a classificação de risco, o acolhimento, a adesão ao tratamento, entra dentro dessa é... educação permanente, são discutidos o processo de trabalho o tempo todo (ENF2).

\section{Discuss Ão}

Os resultados deste estudo demostram a importância dos pilares da ESF como uma forma de trabalhar com DCNT prevalente em nossa sociedade. A evidência desses pilares pode fortalecer a prática integral, resolutiva no cuidado com vista a valorização do modelo e reconhecimento das fragilidades para melhoria da prática assistencial.

Os próprios profissionais compreendem que DCNT pode ser controlada com diferentes formas de cuidado e não apenas com a adesão ao tratamento medicamentoso. A partir desses relatos observou-se que os profissionais reconhecem as ferramentas que podem ser utilizadas para organizarem o fluxo de atendimento e acompanhamento dos usuários portadores de hipertensão, portanto ações de rastreamento, busca ativa e acompanhamento são estratégias fundamentais.

É essencial que as ações de rastreamento se direcionem na busca de pessoas com níveis pressóricos de pré-hipertensão ou superiores, para que seja possível ofertar um tratamento adequado, de forma a prevenir complicações (GUIMARÃES, 2017).

A busca ativa ou rastreamento são estratégias indispensáveis que devem ser realizadas continuamente por profissionais da atenção básica, com o intuito de identificar pessoas com HAS segundo fatores de 
risco, realizar a estratificação de risco e planejamento do cuidado de forma sistematizada (SILVA, 2016). De acordo com os participantes da pesquisa, o rastreamento de pacientes hipertensos é realizado na unidade, no momento em que o mesmo comparece para algum tipo de atendimento, ou seja, livre demanda.

O rastreamento e ações de educação em saúde na comunidade são essenciais para identificar precocemente a HAS na população de abrangência. Se tratando de uma doença que na maioria das pessoas se manifesta de forma assintomática, é necessário ressaltar a importância do rastreamento no controle da HAS e fatores de risco (MENDES, 2016; GUIMARÃES, 2017).

Segundo Mendonça (2015) os grupos são apresentados como forma de controle da HAS e promoção da saúde. Trata-se de uma importante estratégia para trabalhar a educação em saúde na população hipertensa, assim, a educação em saúde através de grupos deve ir além do fornecimento de informações, permitindo a participação ativa e responsabilidade compartilhada dos envolvidos.

Concentrar a participação do usuário no tratamento é uma forma de fortalecer a educação em saúde, gerando mudanças tanto individuais, quanto coletivas, levando em consideração as experiências e conhecimento prévio de todas as pessoas envolvidas. Tal prática leva a reflexão da necessidade de mudança de hábitos e comportamentos dos usuários, bem como a melhoria da qualidade vida (BORGES, 2019).

Mediante a importância de grupos na questão da educação em saúde e participação ativa da população, os participantes deste estudo confirmaram em dois dos relatos a existência de grupos de atividades físicas para comunidade hipertensa, portanto, é de suma importância evidenciar que a atividade física é uma estratégia importante que integra o tratamento não medicamentoso da HAS, que colabora na redução dos níveis pressóricos e redução na classificação do índice de massa corporal.

Os grupos com ações voltadas para os pacientes hipertensos devem se tornar parte das rotinas de trabalho (BORGES, 2019). Eles se destacam como importante estratégia para trabalhar a questão terapêutica e social, através dele os indivíduos conseguem expressar seus sentimentos e emoções, além de permitir diferentes vivências aos participantes, como: realizar atividades em conjunto, o compartilhamento de experiências, a interação social, a comunicação verbal e não verbal (MEDEIROS; COSTA, 2016)

A visita domiciliar dentro da ESF é considerada importante, onde os profissionais, inclusive o enfermeiro, acompanha a família e promove a educação em saúde, reduzindo o risco de adquirir novas doenças. Diante disso, torna-se primordial para as ações desempenhadas na ESF, que por intermédio dos ACS, se forma um elo entre a unidade de saúde e a comunidade, facilitando a ações de promoção da saúde(SANTANA, 2015, MEDEIROS; COSTA, 2016).

Esse elo entre o profissional de saúde que faz a visita e o usuário do serviço é fortalecido pelo vinculo que se estabelece, sendo capaz de favorecer a integralidade da assistência, auxiliando o usuário a desenvolver sua autonomia para tomada de decisões sobre a gestão de seus cuidados (MENDES, 2016).

Além das visitas, o acompanhamento foi destacado pelos profissionais por meio das consultas, médicas ou de enfermagem. Sugere-se que as consultas ocorram de acordo com protocolos assistenciais que embasem suas condutas (SOUZA, 2015).

O acompanhamento desses pacientes é preconizado pelo MS (BRASIL, 2014) para que seja realizado pelo médico e enfermeiro, podendo estes solicitar ajuda de outro profissional quando necessário e as consultas são definidas através da pontuação obtida da Classificação de Framingham.

Quando focada no paciente hipertenso, essas consultas contribuem para a sensibilização sobre sua real situação de saúde, influenciando-o a assumir sua responsabilidade como coparticipante em seu próprio tratamento, de forma a se responsabilizar também pelo cumprimento de metas propostas relacionadas à mudança de comportamento, hábitos alimentares adequados e prática de atividade física (PIMENTA; CALDEIRA, 2014).

As reuniões de equipe permitem o desenvolvimento de uma interação profissional, assim como o 
compartilhamento de responsabilidade sobre as condutas. É através destas que os conhecimentos e responsabilidades são compartilhados e os casos são discutidos (SILVA; MATOS; FRANÇA, 2017), sendo considerada uma proposta de organização e fortalecimento do trabalho em equipe (SILVA; MATOS; FRANÇA, 2017).

Destaca-se que uma das ferramentas que pode ser utilizada nesse momento é o Plano Terapêutico Singular, instrumento de trabalho da clínica ampliada que permite uma abordagem integral por meio do planejamento de ações interdisciplinares e sistematizadas (SANTOS, 2016).

O trabalho em equipe é a base para reorientar o processo de trabalho na APS, além de contribuir para que os princípios do Sistema Único de Saúde sejam atendidos (SANTOS, 2017). A fala dos trabalhadores também ressalta a Educação Permanente em Saúde (EPS) como uma ferramenta para desenvolvimento das atividades assistenciais.

A Política Nacional de Educação Permanente em Saúde é uma estratégia do Sistema Único de Saúde para qualificação e desenvolvimento da capacidade resolutiva dos profissionais no setor de saúde. (SILVA; MATOS; FRANÇA, 2017).

Portanto, essa política ainda define a EPS como aprendizagem no trabalho, onde a capacitação deve levar em consideração as necessidades de saúde da população e da gestão, fortalecendo os profissionais e usuários dos serviços para que possam usufruir de novas formas de assistência, sendo uma alternativa para instrumentalização do processo de trabalho, construindo um novo olhar sobre a dinâmica de assistência (SILVA; MATOS; FRANÇA, 2017).

É importante ressaltar que a EPS tem um conceito próprio, porém muitas vezes os trabalhadores visualizam essa educação como um treinamento. Destaca-se que na EPS podem ocorrer decisões coletivas pelo treinamento ou pela capacitação, porém o foco não é uma abordagem de temas específicos, e sim uma discussão sobre as melhores alternativas para enfrentamento dos desafios durante o processo de trabalho.

Considerando a EPS como modificadora do processo de trabalho, esta ainda colabora com a redução da alienação e burocracia, além de influenciar de forma significativa a capacidade reflexiva dos profissionais, melhorar a questão da autonomia, autoestima e responsabilidade profissional (SILVA; MATOS; FRANÇA, 2017).

Assim, a EPS é um instrumento fortalecedor da gestão, pois permite a reflexão dos trabalhadores a respeito do sentido do cuidado, buscando novos caminhos para garantia de um cuidado integral (BRASIL, 2014).

Os principais resultados evidenciados pelas categorias apresentadas pontuam uma série de atividades que buscam organizar, programar e planejar a assistência ao usuário hipertenso, porém essas ações em sua maioria ocorrem dentro das unidades de saúde, ou seja, o usuário precisa buscar o serviço. A única ação fora da unidade apontada são as visitas. Ao mesmo tempo que há essa limitação, também se faz presente um esforço coletivo em desenvolver outras ações que visam minimizar os desafios identificados no tratamento da hipertensão.

Diante de todos os fatos apresentados, novas práticas de saúde devem ser abordadas para trabalhar as fragilidades presentes no tratamento do usuário hipertenso, de forma a garantir resultados positivos em relação aos indicadores de saúde referentes a essa DCNT.

Frente aos desafios encontrados no processo do cuidado do usuário hipertenso, o tratamento e acompanhamento deve ser feito por uma equipe multiprofissional, de forma longitudinal, mantendo o vinculo e a responsabilidade de todos os membros da equipe, isso pode ocorrer por meio de planos terapêticos individualizados capazes de incentivar o autocuidado apoiado, mantendo o cuidado centrado na pessoa e valorizando os aspectos culturais, comunitários, familiares, assim como a vulnerabilidade do individuo.

Entretanto, este estudo apresentou a limitação da participação de apenas duas categorias profissionais (enfermeiros e Agentes Comunitários de Saúde), o que torna os achados sobre o fluxo de cuidado repre- 
sentativo somente as suas percepções. Ressalta-se que esses profissionais apresentam influência e contato direto no processo de cuidado dos usuários hipertensos.

\section{Conclusão}

Conclui-se que o fluxo de atendimento do usuário hipertenso na ESF não se limita apenas a consultas pontuais ou tratamento medicamentoso, portanto entender como o processo desse cuidado é realizado torna-se essencial para que se valorize as boas práticas assistenciais, assim como pode ajudar a trabalhar com as fragilidades.

Esse conjunto de ações pode favorecer a adesão do usuário hipertenso ao tratamento, assim como contribui para o fortalecimento da ESF e suas ações de gestão, planejamento e enfrentamento dessa DCNT.

\section{REFERÊNCIAS}

BRASIL. Educação permanente em saúde: um movimento instituinte de novas práticas do Ministério da Saúde: agenda 2014. Brasília: Ministério da Saúde; 2014.

Manejo da Hipertensão Arterial Sistêmica e Diabetes Mellitus na Atenção Primária à Saúde. Brasília: Ministério da Saúde; 2018.

. Diretrizes para o cuidado das pessoas com doenças crônicas nas redes de atenção à saúde e nas linhas de cuidado prioritárias. Brasília, DF: 2013.

BORGES, F. M, et al. Health literacy of adults with and without arterial hypertension. Rev Bras Enferm., Brasília, v. 72, n. 3, p. 646-53. 2019.

GUIMARÃES, M. S. A, et al. Estratégia saúde da família e uso racional de medicamentos: o trabalho dos agentes comunitários em Palmas (TO). Trab Educ Saúde., Rio de Janeiro, v. 15, n.1, p. 183-203. 2017.

MENDES, C. R. S, et al. Self-care practice of patients with arterial hypertension in primary health care. Rev Rene., Fortaleza, v. 17 n. 1, p. 52-9. 2016.

MENDES, E. V. A construção social da Atenção Primária à Saúde. Conselho Nacional de Secretários de Saúde - CONASS, 2015. Brasília: Ministério da Saúde; 2015.

MEDEIROS, L. S; COSTA, A. C. M. Postpartum period: the importance of home visits given by the nurse in Primary Health Care. Rev Rene., Fortaleza, v.17, n. 1, p. 112-9. 2016.

MENDONÇA, F. F; NUNES, E. F. P. A avaliação de grupos de educação em saúde para pessoas com doenças crônicas. Trab Educ Saúde., Rio de Janeiro, v. 13, n. 2, p. 397-409. 2015.

MINAYO, M. C. S; DESLANDES, F. P; GOMES, R. Pesquisa Social. Teoria, método e criatividade. Petrópolis: Vozes; 2014.

PAUlA, E. A. et al . Avaliação do risco cardiovascular em hipertensos. Rev. Latino-Am. Enfermagem., Ribeirão Preto, v. 21, n. 3, p. 820-827, jun., 2013. 
PIMENTA, H. B; CALDEIRA, A. P. Fatores de risco cardiovascular do Escore de Framingham entre hipertensos assistidos por equipes de Saúde da Família. Ciênc Saúde Coletiva., Rio de Janeiro, v. 19, n. 6, p.1731-9. 2014.

SANTANA, J. C. B, et al. Visita domiciliar dos agentes comunitários de saúde no planejamento das ações das estratégias da saúde da família: avanços e desafios. Enferm Rev., Belo Horizonte, v. 18 n. 2, p. 18-28. 2015.

SANTOS, E. O, et al. Team meeting: proposal for the work process organization. J Res Fundam Care online., Rio de Janeiro, v. 9, n. 3 p. 606-13. 2017.

SANTOS, R. R, et al. A influência do trabalho em equipe na Atenção Primária à Saúde. Rev Bras Pesq Saúde., Vitória, v.18, n. 1, p.130-9. 2016.

SILVA, K. L; MATOS, J. A. V; FRANÇA, B. D. The construction of permanent education in the process of health work in the state of Minas Gerais, Brazil. Esc Anna Nery., Rio de Janeiro, v. 21, n. 4, p. 2017.

SILVA, R. L. D. T, et al. Evaluation of the care program implementation to people with high blood pressure. Rev Bras Enferm., Brasília, v. 69 n.1 p. 79-87. 2016.

SOUZA, A. S. J, et al. Nursing consultation to hypertensive patients in family health strategy. Rev Enferm UERJ., Rio de Janeiro, v. 23, n. 1, p.102-7. 2015. 